\title{
Bildung von geordneten Konstruktionsfamilien unter Anwendung von Ähnlichkeitsgesetzen
}

\section{P. Gendarz}

Eingegangen: 27. August 2013 / Online publiziert: 18. Oktober 2013

(C) The Author(s) 2013. Dieser Artikel ist auf Springerlink.com mit Open Access verfügbar

Zusammenfassung Die Theorie der Konstruktionsähnlichkeit basiert auf der Theorie der physikalischen Ähnlichkeit. Das primäre Ziel dieser Arbeit ist eine derartige Auswahl von Konstruktionsmerkmalen in den einzelnen Typengrößen der Typenreihen, um identische: physikalische, stereomechanische aber auch einfache Zustände, wie in der Grundkonstruktion, zu erhalten. Die Grundaufzeichnungsform einer Konstruktion einer Typenreihe ist die programmierbare, grafische und relationale Parametrisierung.

\section{Technical means series of types generation process with} constructional similarity theory use Abstract The theory of Constructional Similarity bases on
the theory of physical similarity. The pattern construction
is a model in the theory of constructional similarity. The
essence of this work is to choose such constructional fea-
tures of the new designed constructional means to obtain the
identical states: physical, stereo mechanical or simple like
in the pattern construction.

\section{Formelzeichen}

Formelzeichen, Einheit

$\mathrm{Pa}_{a}^{u} ;(a=1, a z),-$

$x_{i a}^{u}=p a_{i a}^{u},-$

$e_{j}$

\section{Erklärung \\ Parametr der \\ Konstruktionsfamilie \\ Parametermatrix \\ Element des \\ technisches Mittel}

\section{P. Gendarz ( $\square)$}

Institut für Automatisierung und Integrierte Fertigungssysteme, Schlesische Technische Universität, ul. Konarskiego 18a, 44-100 Gliwice, Poland

e-mail: piotr.gendarz@ polsl.pl

$\begin{array}{ll}y_{i l}^{e_{j}}=w_{i l}^{e_{j}}(j=1, j z), \mathrm{mm} & \text { Matrix von Variable } \\ \bar{X}_{0}\left\{x_{0 a} ;(a=1, a z)\right\},- & \text { Abmessungswerten } \\ k s_{0}\left\{y_{o l}^{e_{j}} ;\left(1=1, l v_{j}\right)(j=1, j z)\right\},- & \text { Musterparametern } \\ \bar{X}_{i}^{u}\left\{x_{i a}^{u} ;(i=1, i z)(a=1, a z)\right\},- & \text { Musterparametern } \\ k s_{i}\left\{y_{i l}^{e_{j}} ;\left(1=1, l v_{j}\right)(j=1, j z)\right\} & \text { Neue Parametern } \\ \quad \in \mathrm{RK}_{n} & \text { Neue Konstruktion } \\ \mathrm{Po}_{n}\left\{p o_{i} ;(i=1, i z)\right\},- & \text { Sammlung von } \\ t k s_{m}^{z_{n}},- & \text { Bedürfnissen } \\ & \text { Typische } \\ \varphi_{a}^{u},- & \text { Konstruktionslösung } \\ \varphi_{1}^{e_{j}},- & \text { Parameterähnlich- } \\ & \text { keitszahl } \\ \operatorname{Re}_{I}^{z_{r}},- & \text { Abmessungsähnlich- } \\ \sigma, \mathrm{MPa} & \text { keitszahl } \\ F, \mathrm{~N} & \text { Isomorphische } \\ A, \mathrm{~mm}{ }^{2} & \text { Relation des Systems } \\ \left\langle x_{n}, y_{n}\right\rangle,- & \text { Normalspannung } \\ & \text { Kraft } \\ p b,- & \text { Querschnittsfläche } \\ & \text { Koordinaten der } \\ & \text { charakteristischen } \\ & \text { Konturpunkte } \\ & \text { Basispunkt } \\ & \end{array}$

\section{Einführung}

Das praktische Ziel der Theorie der Konstruktionsähnlichkeit ist die Bildung von geordneten Konstruktionsfamilien. Ein Beispiel ist die Bildung einer Konstruktions-Typenreihe, die auf einer Musterkonstruktion und gemeinsamen vereinheitlichten Parametern basiert (siehe Abb. 1).

Neue Herausforderungen im Konstruktionsprozess gehen nicht nur in Richtung der Entwicklung neuartiger Konstruk- 
Abb. 1

Konstruktions-Typenreihe gebildet auf der Basis einer Musterkonstruktion

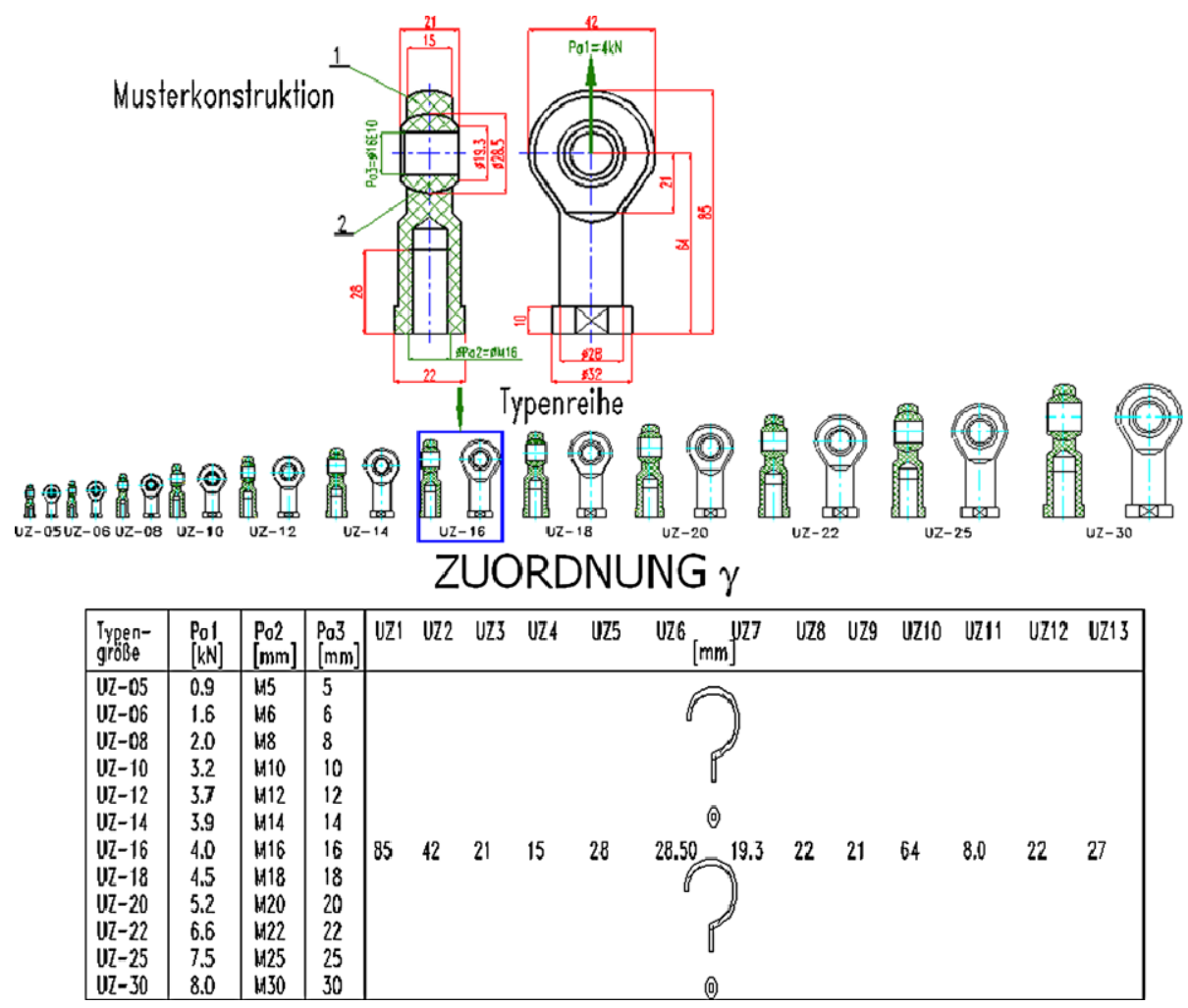

tionslösungen, sondern auch in Richtung ein größtmögliches Spektrum von Bedürfnissen für eine bestimmte Art von technischen Mitteln zu umfassen [2].

Nehmen wir zum Beispiel die Herstellung von Ösen, die eine Last von $\mathrm{Pa} 1=4 \mathrm{kN}$ übertragen sollen und einen Durchmesser der Stange oder des Kolbens von Pa2 $=$ M16 sowie einen Durchmesser der Ösenöffnung von Pa3 = $16 \mathrm{~mm}$ besitzen sollen. Aus marktwirtschaftlichen Gründen würde ein solches Produkt nur auf beschränktes Interesse stoßen. Wird hingegen eine geordnete Konstruktionsfamilie in Form einer Ösen-Typenreihe für die Bedürfnissphäre gebildet, die durch vereinheitlichte Parameter mit den Wertebereichen Pa1 $=0,9-8 \mathrm{kN}, \mathrm{Pa} 2=\mathrm{M} 5-\mathrm{M} 30 \mathrm{~mm}$ und $\mathrm{Pa} 3=5-30 \mathrm{~mm}$ gegeben seien, so gewinnt diese viel mehr an Aufträgen und entspricht besser den Anforderungen des Kunden, wodurch auch bessere Wettbewerbsfähigkeit am Markt vorliegt. Somit ist die Entwicklung von Konstruktionsmethoden, die auf der Grundlage einer einzigen Konstruktion eine rationale Typenreihe mit einer konstanten Gestalt, aber mit veränderlichen Abmessungen aufbauen, begründet. Basierend auf dieser Grundlage läuft die QualitätQuantität Fragenstellung auf eine Quantität Fragenstellung hinaus, d.h. für welche vereinheitlichten Parameter $p a_{i a}^{u}$ optimale Abmessungswerte ausgewählt werden sollen $w_{i l}^{e_{j}}$ $(j=1, j z)$. Die Zuordnung im Prozess der Bildung von geordneten Konstruktionsfamilien (Typenreihen, Modulsys- temen) wird als Zuordnung $\gamma[1,3]$ bezeichnet.

$p a_{i a}^{u} \rightarrow w_{i l}^{e_{j}} \quad(j=1, j z)$.

Die Methode der Bildung einer geordneten Konstruktionsfamilie auf der Basis der Konstruktionsähnlichkeit unterscheidet folgende Phasen:

- Auswahl, Überprüfung und Änderung der Musterkonstruktion,

- Vereinheitlichen der Parameter für eine geordnete Familienkonstruktion,

- Aufzeichnung der Konstruktion mit variabler Abmessungsanordnung,

- Schaffung von Voraussetzungen für die Ähnlichkeit von Parametern und Abmessungen,

- Berechnung und Überprüfung der Abmessungswerte von Element-Typenreihen,

- parametrische Konstruktionsaufzeichnung.

\section{Musterkonstruktion}

Die Musterkonstruktion $\left(k s_{0}\right)$ ist eine bereits überprüfte Konstruktion im Hinblick auf die Funktion (Funktionssimulation mittels CAD), Optimierung (Festigkeitsanalyse mittels CAE) und Fertigung (Fertigungssimulation mittels CAM). Eine Musterkonstruktion ist auch bereits experimentell untersucht worden (Prototypuntersuchungen). Ei- 
Abb. 2 Bildung von Musterkonstruktionen für hydraulische Zylinder

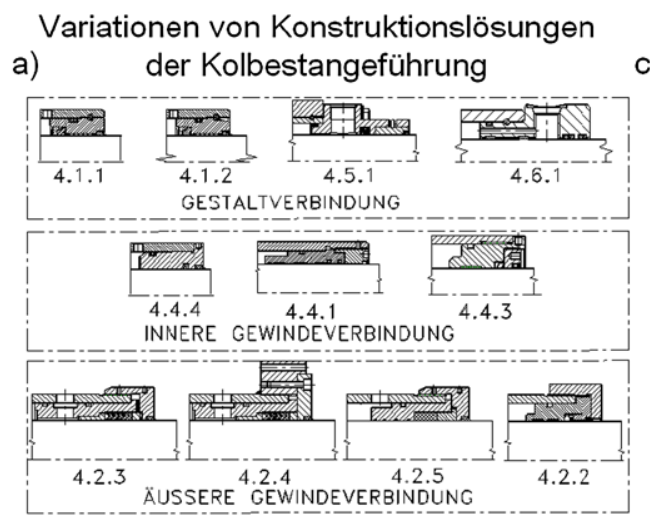

Musterkonstruktionen von hydraulischen

c) Zylindern für Bergbaumaschinen
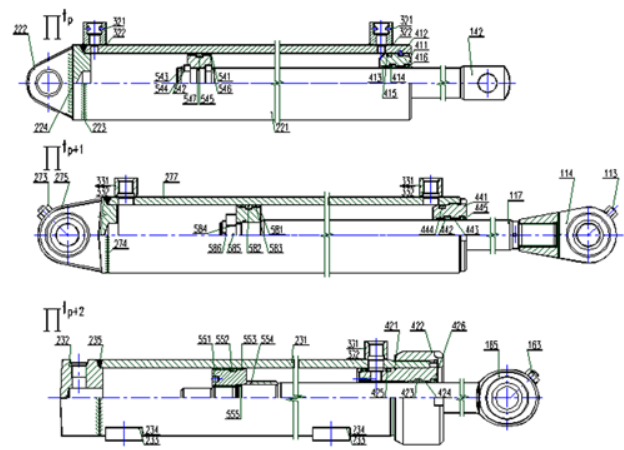

Bewertung und Auswahl
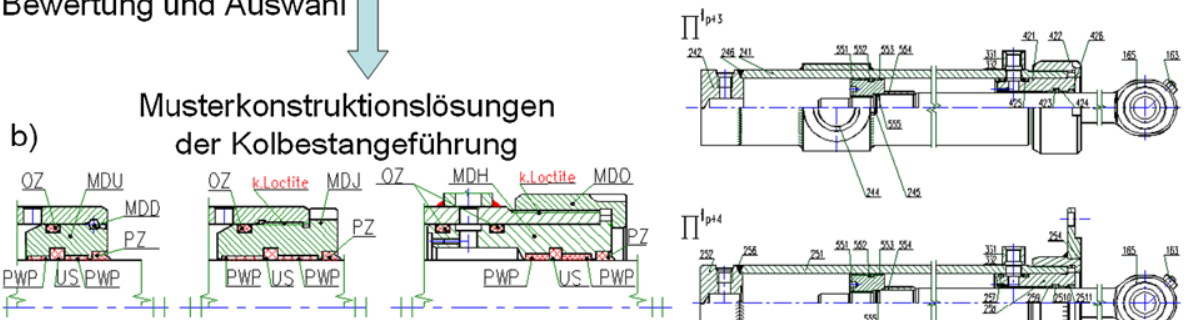

Musterkonstruktionslösungen der Kolbestangeführung

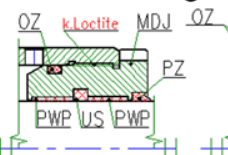

$$
4.1 .0
$$

4.4 .0
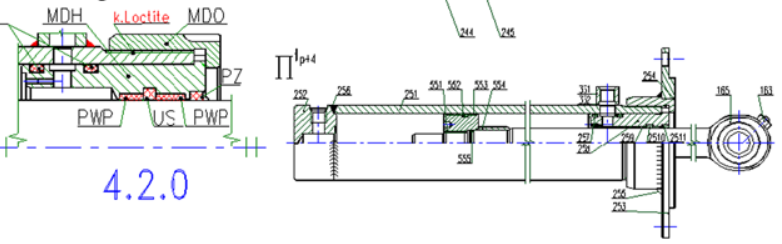

ne Konstruktion kann als eine Musterkonstruktion angenommen werden, wenn sie die Kriterien für eine experimentelle Untersuchung bestmöglich erfüllt. Aufgrund der Berechnungsgenauigkeit der Abmessungswerte mittels der Methode der Konstruktionsähnlichkeit, wird als Musterkonstruktion eine Konstruktion aus dem mittleren Bereich der geordneten Parameter empfohlen. Es wird zwischen zwei Erstellungsmöglichkeiten für Musterkonstruktionen unterschieden:

1. Durch Auswahl aus einer existierenden Konstruktionsmenge, die bereits eine Produktionsbasis bildet und deren hergestellte Anwendungsanforderungen erfüllt,

2. Anwendung der Variantenanalyse für eine neu zu erstellende Musterkonstruktion.

Im ersten Fall sind die Hauptziele der Musterkonstruktionsauswahl die Erweiterung des Angebots (des Bedarfs) einer bereits gefertigten Klasse von technischen Objekten. Zunächst wird eine praktische Beurteilung der bestehenden Konstruktionslösungen durch verschiedene Experten durchgeführt: Planer, Konstrukteure, Technologen, Ökonomen, Hersteller, Anwender und Instandhaltungs-Techniker. Auf dieser Grundlage wird die optimale Wahl getroffen, eventuell eine Konstruktionsmodifikation durchgeführt und somit die Musterkonstruktion $k s_{0}$ gebildet. Ein Beispiel sind ausgewählte Musterkonstruktionen von Kolbestangeführungen, die in hydraulischen Zylindern für Bergbaumaschinen angewendet werden (Abb. 2a). Das Ergebnis der Bewertung und Auswahl sind Musterkonstruktionslösungen der Kolbenstangenführungen (Abb. 2b). Durch den Einsatz der glei- chen Verfahrensweise für die restlichen Baugruppen des hydraulischen Zylinders wurden Musterkonstruktionen abgeleitet, wie in Abb. 2c dargestellt.

Ein besonderes Werkzeug zur Bildung von Musterkonstruktionen für eine neu zu erstellende, geordnete Konstruktionsfamilie (vorlaufende Ordnung) ist der Einsatz der Variantenanalyse [5], die beispielsweise im I-DEAS Programm entwickelt wird. Bei der Variantenanalyse wird die Methode der finiten Elemente angewendet die zusätzlich um folgendes erweitert ist:

- Sensitivitätsanalyse - es werden relevante ElementAbmessungen im Hinblick auf den Spannungs- und Verzerrungszustand sowie auch auf die Masse ausgewählt.

- parametrische Analyse - für vorgegebene Bereiche der relevanten Abmessungswerte werden optimale Werte bestimmt [5] im Hinblick auf zulässige Spannungen, Verschiebungen, Verformungen und minimale Masse.

Ein Beispiel für die Anwendung der Variantenanalyse ist die Bildung einer Musterkonstruktion von Greifbacken eines Greifers. Das Ausgangsmodell wird in Abb. 3a dargestellt. Anhand der Ergebnisse der Variantenanalyse (Abb. 3b und $3 \mathrm{c}$ ) wird eine optimale Musterkonstruktion erhalten (Abb. 3d).

In der Musterkonstruktion werden höhere Spannungen zugelassen, die die kritische Spannung, die Streckgrenze nicht überschritten wird. Somit kann infolge der höheren Materialausschöpfung die Elementmasse um $65 \%$ reduziert werden. 
a)

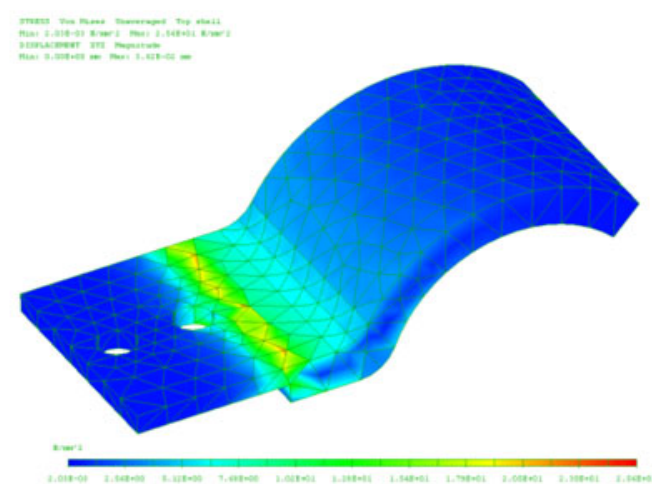

VOR DER OPTIMIERUNG $\sigma=25,6[\mathrm{MPa}]$

c)
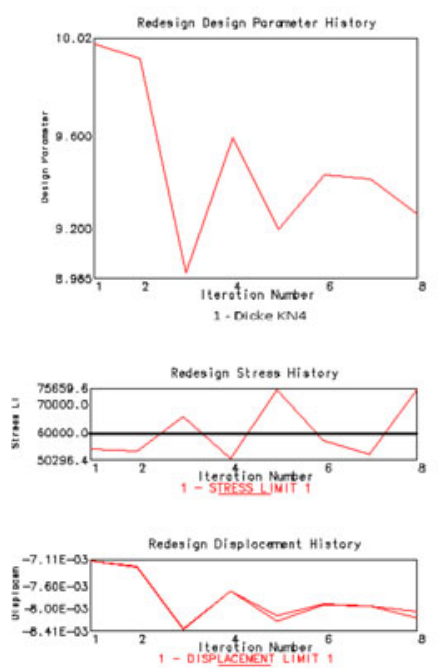
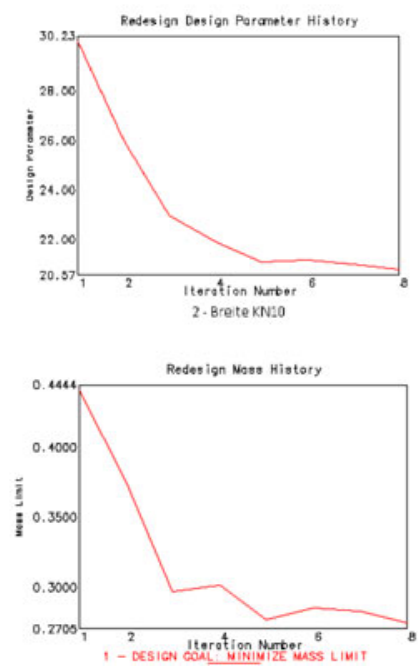

b)

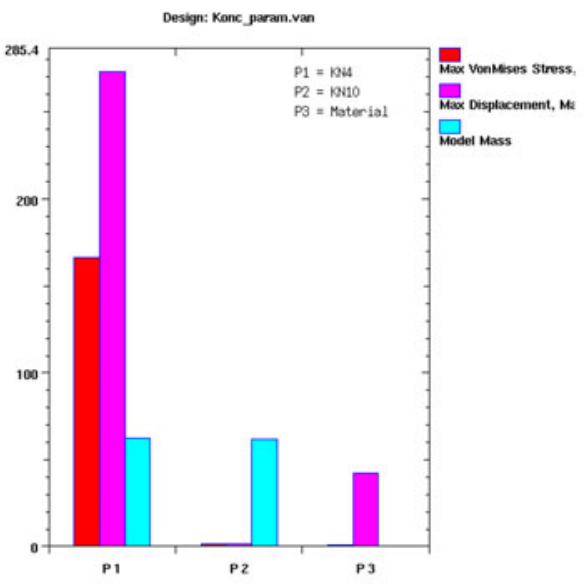

d)

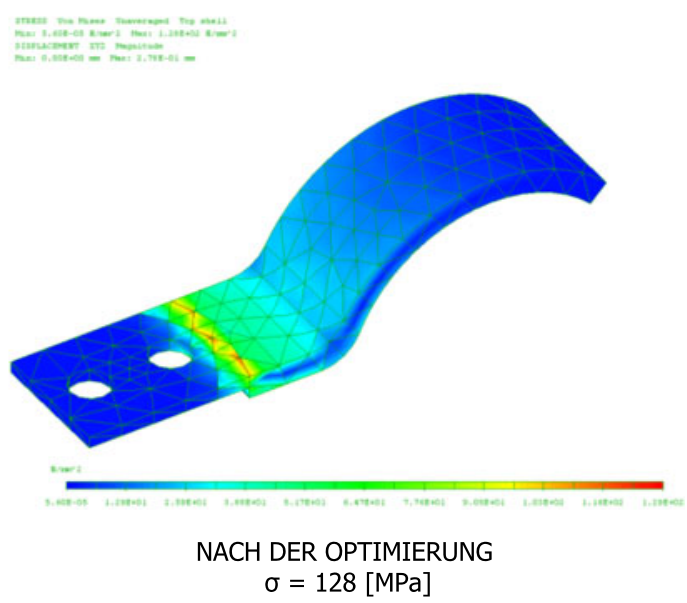

Abb. 3 Variantenanalyse von Greifbacken eines Greifers

\section{Vereinheitlichung und Aufzeichnung von Konstruktionen mit einer Variablenabmessungsanordnung}

Quantitative, charakteristische Familienkonstruktions-Merkmale werden als Parameter $\mathrm{Pa}_{a}^{u} ;(a=1, a z)$ bezeichnet. Die Elemente der Parametermatrix $p a_{i a}^{u}$ sind unabhängige Variable bei der Bestimmung der quantitativen Konstruktionsmerkmale von Elementen $x_{i a}^{u}=p a_{i a}^{u}[1,4]$. Die Auswahl der Konstruktionsmerkmale von Elementen wird aufgrund des Bedürfnisses $p o_{i}^{u}$ vorgenommen, das durch die Zeile der Parametermatrix repräsentiert wird, die aus sogenannten vereinheitlichten Parametern gebildet wird.

Aufgrund der Parameterwerte werden quantitative Konstruktionsmerkmale (Abmessungswerte und Materialkennwerte) der einzelnen hergestellten Elemente $e_{j} y_{i l}^{e_{j}} \quad(l=$ $\left.1, l v_{j}\right)$ ausgewählt. Die Bedürfnissphäre der Konstruktionsfamilie wird durch vereinheitlichte Parameterwerte, also durch beschränkte und geordnete Parameterwerte, die über eine bestimmte Zeit angenommen werden, beschrieben. Die
Werkzeuge zur Generierung von vereinheitlichten Parametern sind: Prognose, Anpassung der Parameterwerte an eine Normalreihe, Adaptation der Parameter an zusammenwirkende Parameter von technischen Objekten [6, 7]. Ein Beispiel für vereinheitlichte Parameter eines Hydraulikzylinders, als unabhängige Variablen der Zuordnung $\gamma$, zeigt die Abb. 4. Der Zuordnung $\gamma$ geht die Zuordnung $\beta$ voraus, die auf der Zerlegung der typischen Konstruktionslösungen beruht und typische Element-Konstruktionsformen mit Abmessungsanordnung generiert. In der Abmessungsanordnung werden die variablen Abmessungen durch Buchstaben und Nummern bezeichnet. Zum Beispiel: Für das Element MTG heißen die Bezeichnungen TG1-TG15 (Abb. 4).

\section{Bedingungen der Konstruktionsähnlichkeit}

Die Methode zur Auswahl von Abmessungswerten auf der Basis der Konstruktionsähnlichkeit hat ihre Ursprünge in der Theorie der physikalischen Ähnlichkeit [7]. Die physischen Modelle werden in einem entsprechenden Maßstab 
Abb. 4 Zuordnung $\boldsymbol{\beta}$ und $\boldsymbol{\gamma}$

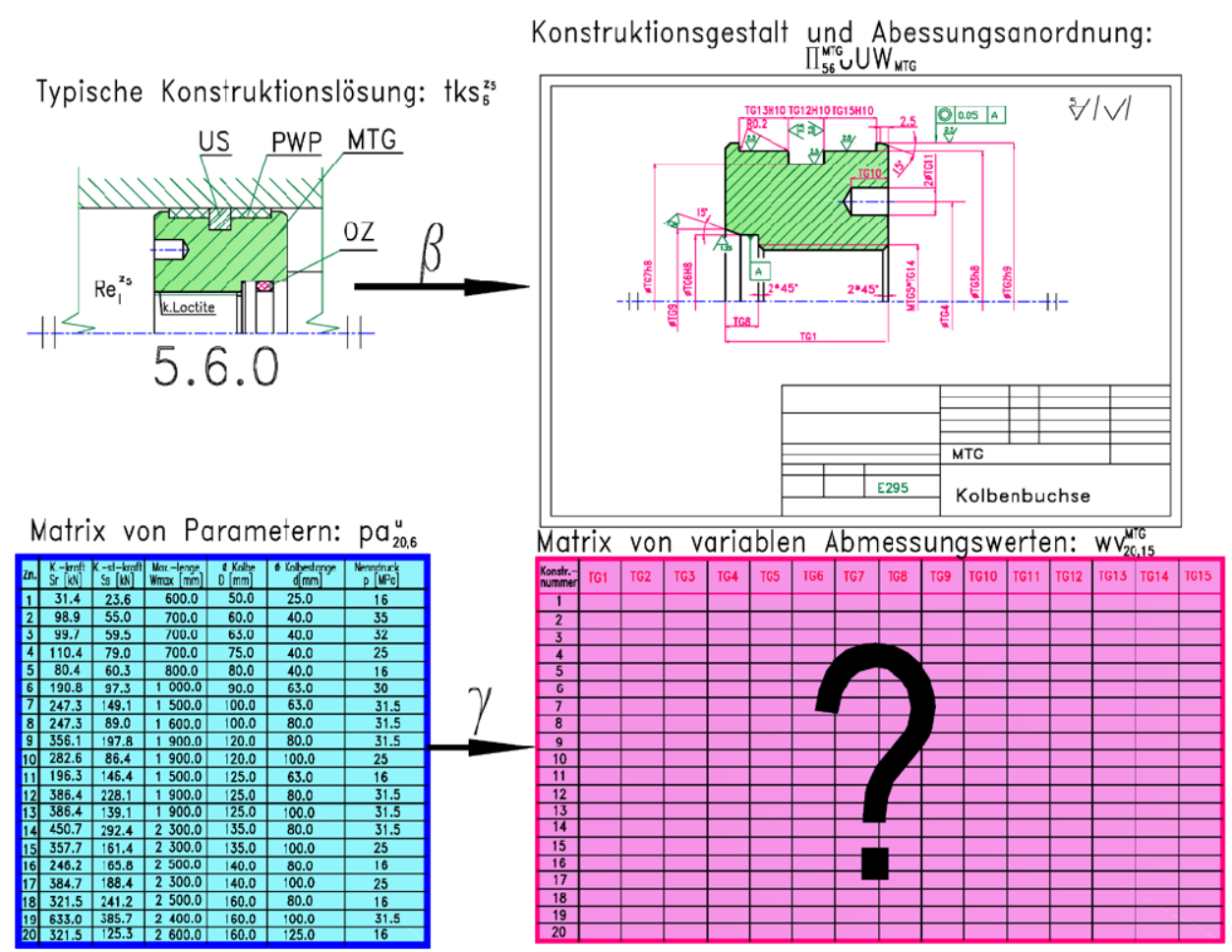

Abb. 5 Modell für quantitative Wahl von

Konstruktionsmerkmalen basierend auf der Konstruktionsähnlichkeit

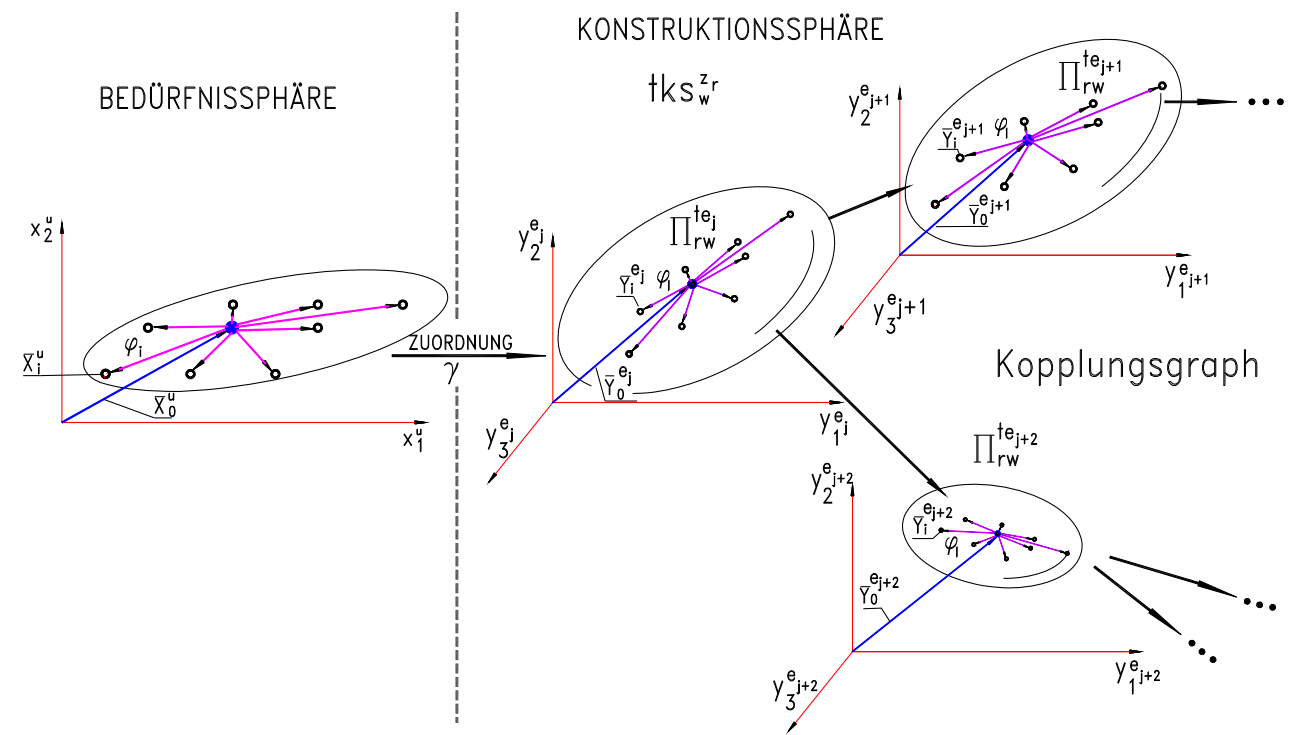

erstellt und getestet, so dass komplexe physikalische Phänomene gut simuliert werden können. Auf dieser Grundlage werden neue Konstruktionen technischer Objekte geschaffen. Zum Beispiel wird für ein Flugzeugmodell im entsprechenden Maßstab in einem Windkanal die geometrische Form des Rumpfs und seine Abmessungsverhältnisse modifiziert. Im Prozess der Generierung von geordneten Konstruktionsfamilien mit der Anwendung der Konstruktionsähnlichkeit entspricht dem Modell: die Musterkonstruk- tion $k s_{0}\left\{y_{o l}^{e_{j}} ;\left(l=1, l v_{j}\right)(j=1, j z)\right\}$ mit den Musterparametern $\bar{X}_{0}\left\{x_{0 a} ;(a=1, a z)\right\}$. Auf ihrer Basis werden geometrisch ähnliche Konstruktionen geschaffen $k s_{i}\left\{y_{i l}^{e_{j}} ;(l=\right.$ $\left.\left.1, l v_{j}\right)(j=1, j z)\right\} \in \mathrm{RK}_{n}$, entsprechend zu den unifizierten Parametern $\bar{X}_{i}^{u}\left\{x_{i a}^{u} ;(i=1, i z)(a=1, a z)\right\}$, die in diesen identische Relationskopplungen und Umwandlungsrelationen erfüllen (Abb. 5).

Es werden zwei grundlegende Ähnlichkeitszahlen zwischen den Konstruktionsmerkmalen der neuen Konstruktion 

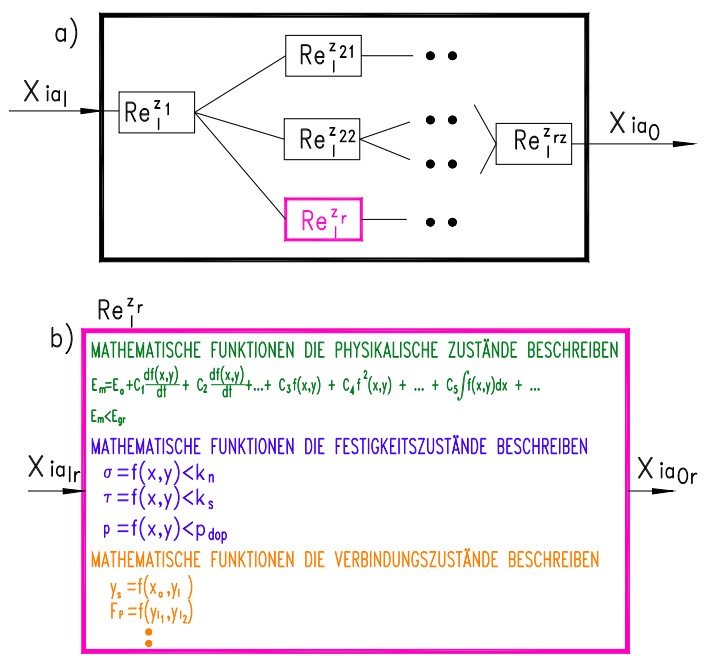

Abb. 6 Mathematische Beschreibung der zukünftigen Zustände eines technischen Mittels

$k s_{i}$ und der Musterkonstruktion $k s_{0}$ definiert:

Parameterähnlichkeitszahl $\quad \varphi_{a}^{u}=\frac{x_{i a}^{u}}{x_{0 a}}$,

Abmessungsähnlichkeitszahl $\quad \varphi_{l}^{e_{j}}=\frac{y_{i l}^{e_{j}}}{y_{0 l}^{e_{j}}}$.

In der Theorie der Konstruktionsähnlichkeit werden die Konstruktionsmerkmale gewählt, um Relationskopplungen und Umwandlungsrelationen des Systems in einer neuen Konstruktion zu erhalten (Abb. 6a), die denen der Musterkonstruktion entsprechen und somit optimal unterschiedliche Konstruktionsmengen bilden. Isomorphen Relationskopplungen und Umwandlungsrelationen, die in der Systemstruktur von Konstruktionsfamilien hervorgehoben sind, werden phänomenologische Modelle und physikalische Abhängigkeiten, die mit mathematischen Funktionen beschrieben werden, zugeordnet (Abb. 6b).

Werden identische Zustände beibehalten: physikalische, stereomechanische und einfache, beschrieben jeweils mittels mathematischer Funktionen für einzelne Relationen der Systemstruktur der Konstruktionsfamilie, so werden die Bedingungen der Konstruktionsähnlichkeit gebildet [7]. Diese Bedingungen werden als Grundlage für die Berechnung der Konstruktionsmerkmale $y_{i l}^{t e_{j}}\left(l=1, l v_{i s}\right)=$ $f_{p}\left(x_{i a}^{u}\right)$ herangezogen. Die Werte der Konstruktionsähnlichkeit für die folgenden, angenommenen Ähnlichkeitszahlen: Kraft $\varphi_{F}$, Drehmoment $\varphi_{M o}^{u}$, Fläche $\varphi_{A}$, Volumen $\varphi_{V}$, Widerstandsmoment $\varphi_{W}$, Massenträgheitsmoment $\varphi_{J}$, etc. werden in der Funktion der Abmessungsähnlichkeit $\varphi_{l}$ bestimmt. Hingegen betreffen die Konstruktionsähnlichkeitszahlen $\varphi_{l}$ die quantitativen Konstruktionsmerkmale bei der Voraussetzung einer konstanten Konstruktionsgestalt $\Pi$ [3]. Für die angenommene Ähnlichkeit der
Tab. 1 Grundsätzliche Beziehungen zwischen Ähnlichkeitszahlen [7]

\begin{tabular}{lll}
\hline Größe & Bezeichnung & Ähnlichkeitsmaßstab \\
\hline Drehzahl & $\varphi_{n}$, & $\varphi_{l}^{-1}$ \\
Winkelgeschwindigkeit & $\varphi_{\omega}$ & \\
Geschwindigkeit & $\varphi_{\nu}$, & $\varphi_{l}^{0}=1$ \\
Infolge Elastischer- und & $\varphi_{\varepsilon}$, & \\
Trägheits-Kräfte: & & \\
Dehnung & $\varphi_{\sigma}, \varphi_{\mathrm{p}}$ & \\
Spannung, & & \\
Flächenpressung & $\varphi_{\Delta \mathrm{l}}$, \\
Elastische Verformung, & $\varphi_{l}^{1}$ \\
Federsteifigkeit & $\varphi_{\varepsilon}$, \\
Infolge Schwerkraft: & $\varphi_{\sigma}, \varphi_{\mathrm{p}}$ & \\
Dehnung, Spannung, & & \\
Flächenpressung & & \\
Kraft & $\varphi_{\mathrm{F}}$ & \\
Fläche & $\varphi_{\mathrm{A}}$ & \\
Volumen & $\varphi_{\mathrm{V}}$ & $\varphi_{l}^{2}$ \\
Gewichtskraft & $\varphi_{\mathrm{G}}$, & \\
Drehmoment & $\varphi_{M s}$, & \\
Widerstandsmomente & $\varphi_{W x}, \varphi_{W 0}$ \\
Flächenträgheitsmomente & $\varphi_{I x}, \varphi_{I 0}$ \\
Massenträgheitsmomente & $\varphi_{J x}$ & $\varphi_{l}^{4}$ \\
\hline & &
\end{tabular}

Querschnittsfläche $\varphi_{A}$, z. Bsp. eines Quadrates der Seitenlänge $l$, mit einer Seitenlängenähnlichkeit $\varphi_{l}$ ergibt sich:

$A=l^{2}, \quad \varphi_{A}=\left(\frac{l_{i}}{l_{0}}\right)^{2}=\varphi_{l}^{2}$.

Der Zusammenhang in (4) gilt auch für andere Querschnitte: Rechteck, Ellipse, etc., vorausgesetzt, dass alle Querschnittsabmessungen gleichmäßig wachsen also einer vollen Abmessungsähnlichkeit entsprechen. Die Bedingung der Ähnlichkeit der Querschnittsfläche bedeutet, dass zum Beispiel eine zweifache Änderung eines Wellendurchmessers $\varphi_{l}=2$ eine vierfache Erhöhung des Querschnittes bewirkt, $\varphi_{A}=4$. Verallgemeinernd werden folgende Beziehungen unterschieden (s. Tab. 1).

Der Grundzustand einer neuen Konstruktion, der der Musterkonstruktion gleichen sollte, ist der stereomechanische Zustand, wo identische Spannungen $\varphi_{\sigma}=1$ herrschen. In Anbetracht einer einfachen Zugspannung in einem Element, hervorgerufen durch die statische Kraft $F$ (ohne Eigengewicht), wird die Spannung aus dem folgenden Verhältnis bestimmt [7]:

$\sigma=\frac{F}{A} \leq \sigma_{d o p}$ 
Abb. 7 Ergebnis der Zuordnung $\gamma$

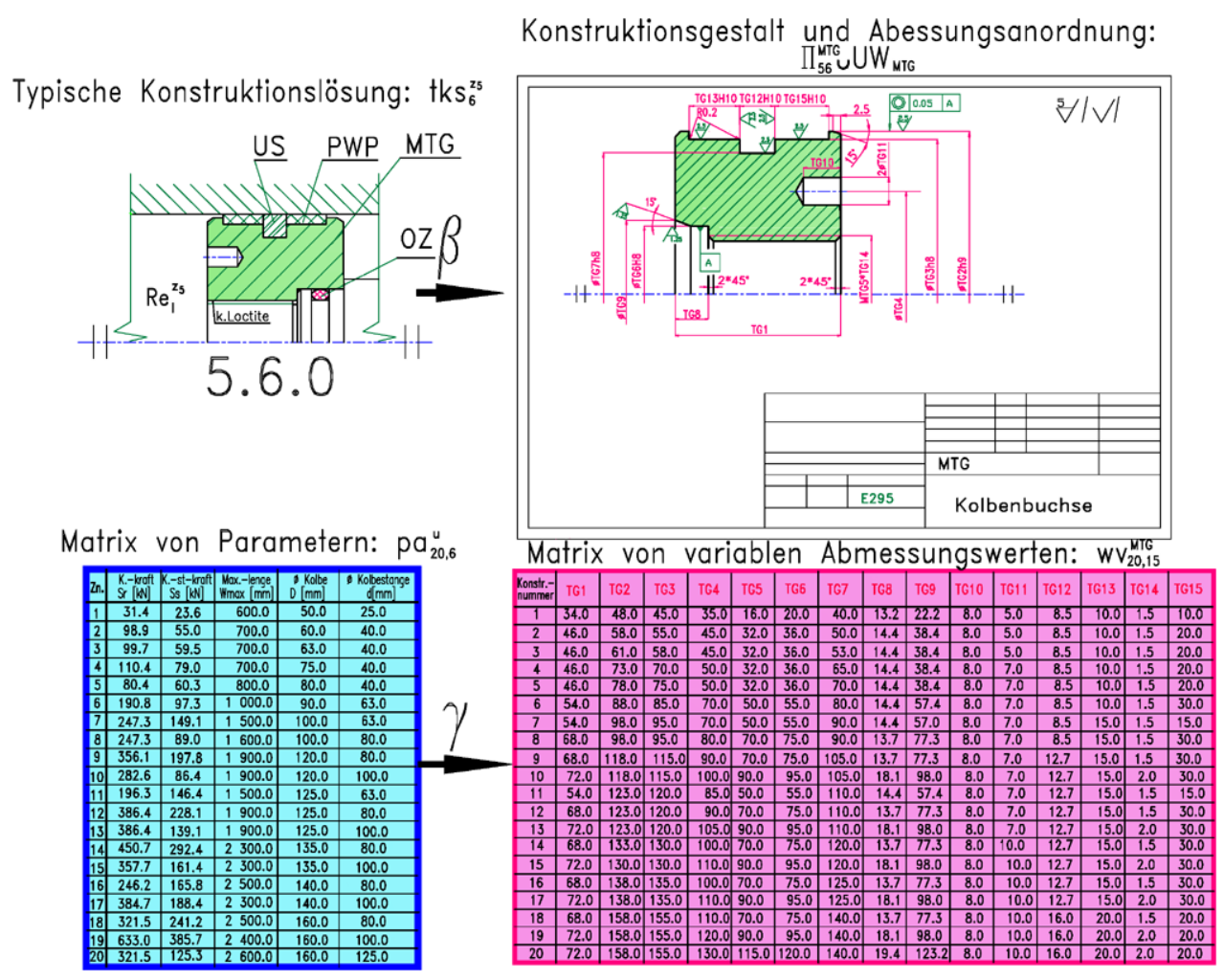

Die Spannungsähnlichkeitszahl in der Typenreihe (Modulreihe) beträgt,

$\varphi_{\sigma}=\frac{\sigma_{i}}{\sigma_{0}}=\frac{F_{i} \cdot A_{o}}{F_{0} \cdot A_{i}}=\frac{\varphi_{F}}{\varphi_{A}}=\frac{\varphi_{F}}{\varphi_{l}^{2}}=1$,

daraus folgt,

$\varphi_{F}=\varphi_{l}^{2}$.

Die Parameterwerte wie auch die Werte der quantitativen Konstruktionsmerkmale der Elemente, auf der Grundlage der Bedingungen der Konstruktionsähnlichkeit, können über folgende Beziehungen berechnet werden:

vereinheitlichte Parameterwerte: $x_{i a}=x_{0 a} \cdot\left(\varphi_{i 1}^{u}\right)^{i}$,

Abmessungswerte: $\quad y_{i l}^{e_{j}}=y_{0 l}^{e_{j}} \cdot\left(\varphi_{i l}^{e_{j}}\right)^{i}$,

Wobei gilt: $x_{0 a}-$ a-ter Musterparameterwert; $y_{0 l}-1$-ter Abmessungswert der Musterkonstruktion; $i$ - Exponentenwert der die Entfernung von der Musterkonstruktion beschreibt; $(i=\ldots-2,-1,0,1,2 \ldots, i=0$ - entspricht der Musterkonstruktion).

Die Parameter- und Abmessungswerte können, wie oben gezeigt, analytisch berechnet bzw. mittels Nomogrammen mit einer logarithmischen Koordinatenskala grafisch bestimmt werden. Das Ergebnis bilden die Abmessungswerte, die auf der Grundlage der Konstruktionsähnlichkeit gewonnen werden und zusätzlich modifiziert sind auf Basis der
Anpassung an die Abmessungen von Katalog- und Normteilen. Beispiele sind die Abmessungswerte einer Kolbenbuchse, gezeigt in der Abb. 7.

\section{Parametrische Konstruktionsaufzeichnung}

Die Parametrisierung ist eine spezielle Form der Konstruktionsaufzeichnung, adaptiert an die Aufzeichnung von Typenreihen einer Konstruktion (Modulreihe), die sich durch eine gleichbleibende Konstruktionsform $\Pi$ und durch veränderliche Abmessungswerte $W$ charakterisieren. In grafischen Programmen wird zwischen dynamischer, relationaler, programmierbarer und grafischer Parametrisierung unterschieden. Im Prozess der Bildung von geordneten Konstruktionsfamilien, mit der Methode der Konstruktionsähnlichkeit, konnten die besten Ergebnisse bei der programmierbaren, grafischen und relationalen Parametrisierung erreicht werden.

Programmierbare Parametrisierung nutzt eine Programmiersprache und ein Grafikprogramm, durch welches diese Sprache angewendet. Beispiele sind die Programmiersprache AutoLISP im grafischen Programm AutoCAD oder die Programmiersprache GRIP im fortgeschrittenen Grafikprogramm NX. Die Aufzeichnung der Elementkonstruktion läuft auf die Bildung einer Parametrik in der AutoLISPSprache hinaus (Abb. 8c), in der Berechnungen hinsichtlich der Bestimmung von Abmessungswerten, mit der Anwen- 


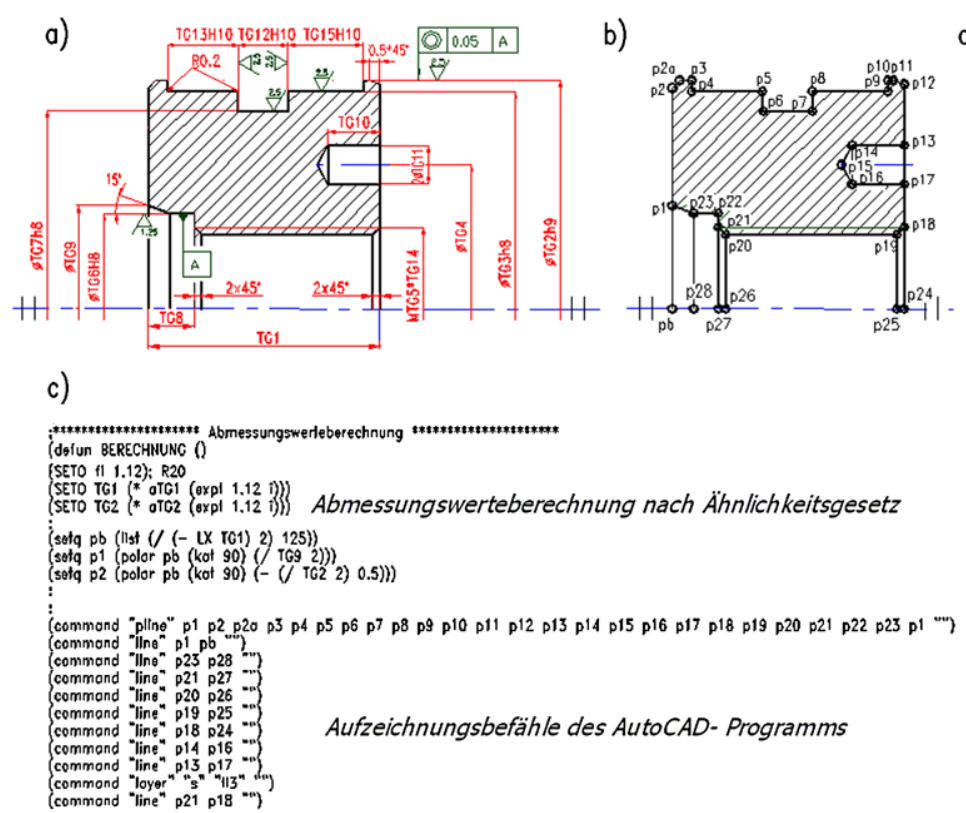

d)

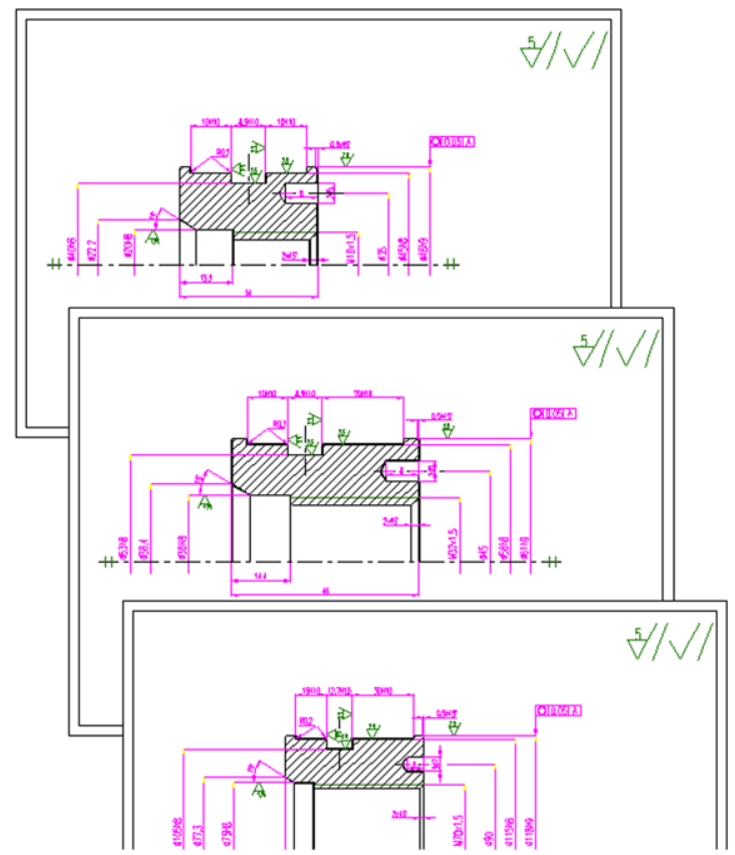

Abb. 8 Aufzeichnung eines MTG-Element-Grundrisses in einer Parametrik

dung der Bedingungen der Konstruktionsähnlichkeit, unternommen werden. Anschließend, durch die Nutzung der Befehle des graphischen Programms, wird eine Konstruktionsaufzeichnung, basierend auf den Koordinaten der charakteristischen Konturpunkte, gebildet (Abb. 8b). Diese Koordinaten werden mittels der Funktion der variablen Abmessungen $T G_{l}$ (Abb. 8a) beschrieben. Allgemein kann geschrieben werden:

$\left\langle x_{n}, y_{n}\right\rangle=f\left(y_{i l}^{t e_{j}}\right)$.

Die Berechnung der Punktekoordinaten beginnt mit dem sogenannten Basispunkt $p b$, (Abb. 8b). Wenn auf der Zeichnung mehrere Projektionen (Ansichten, Teilansichten, Schnittansichten, Teilschnittansichten, Detailansichten) dargestellt werden, dann sind auch mehrere Basispunkte vorhanden. Die Basispunkte beschreiben die Anordnung der Projektionen auf dem Zeichnungsblatt und können durch die Verwendung des angenommenen Zeichenblattformates und des Maßstabes bestimmt werden. Ausgehend vom Basispunkt werden die Koordinaten der charakteristischen Punkte der Aufzeichnung der gegebenen Projektion mittels der Abmessungsfunktion Punkt für Punkt bestimmt (Abb. 8c).

Wird die Parametrik im grafischen Programm ausgeführt, so wird eine Aufzeichnung der Elementkonstruktion für eine ausgewählte Typengröße in Form einer Fertigungszeichnung generiert (Abb. 8d).

In der grafischen Parametrisierung ist die Kenntnis einer Programmiersprache nicht erforderlich, es werden die
Abb. 9 3D-Modell der Musterkonstruktion

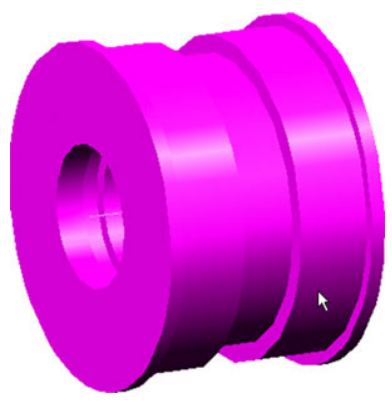

entsprechenden Möglichkeiten des graphischen Programms genutzt. Eine derartige Form der Parametrisierung findet in graphischen Programmen, wie I-DEAS NX oder Unigraphics NX (NX), Anwendung. Zunächst wird hier ein 3DModell der Musterkonstruktion erstellt (Abb. 9).

Während der Bildung eines 3D-Modells werden Abmessungswerte gewählt, die für ein Mustermodell optimal sein sollten. Jedes Abmaß erhält einen Identifizierungscode, der aus Buchstaben (in der Regel aus zwei Buchstaben) und Nummern besteht. Die Reihenfolge in der die Nummern vergeben werden, sollte mit den Gesamtabmessungen beginnen, anschließend mit den gekoppelten (meistens mit Toleranzen) und den restlichen Abmessungen fortgesetzt werden. Für ein auf diese Weise vorbereitetes Mustermodell werden die Möglichkeiten des Grafikprogramms genutzt. Im fortgeschrittenen Grafikprogramm I-DEAS NX wird mit der Auswahl der „Catalog“ Schaltfläche begonnen (Abb. 10a) in der aus der Abmessungsmenge variable Abmessungen 
Abb. 10 Auswahl von variablen Abmessungen

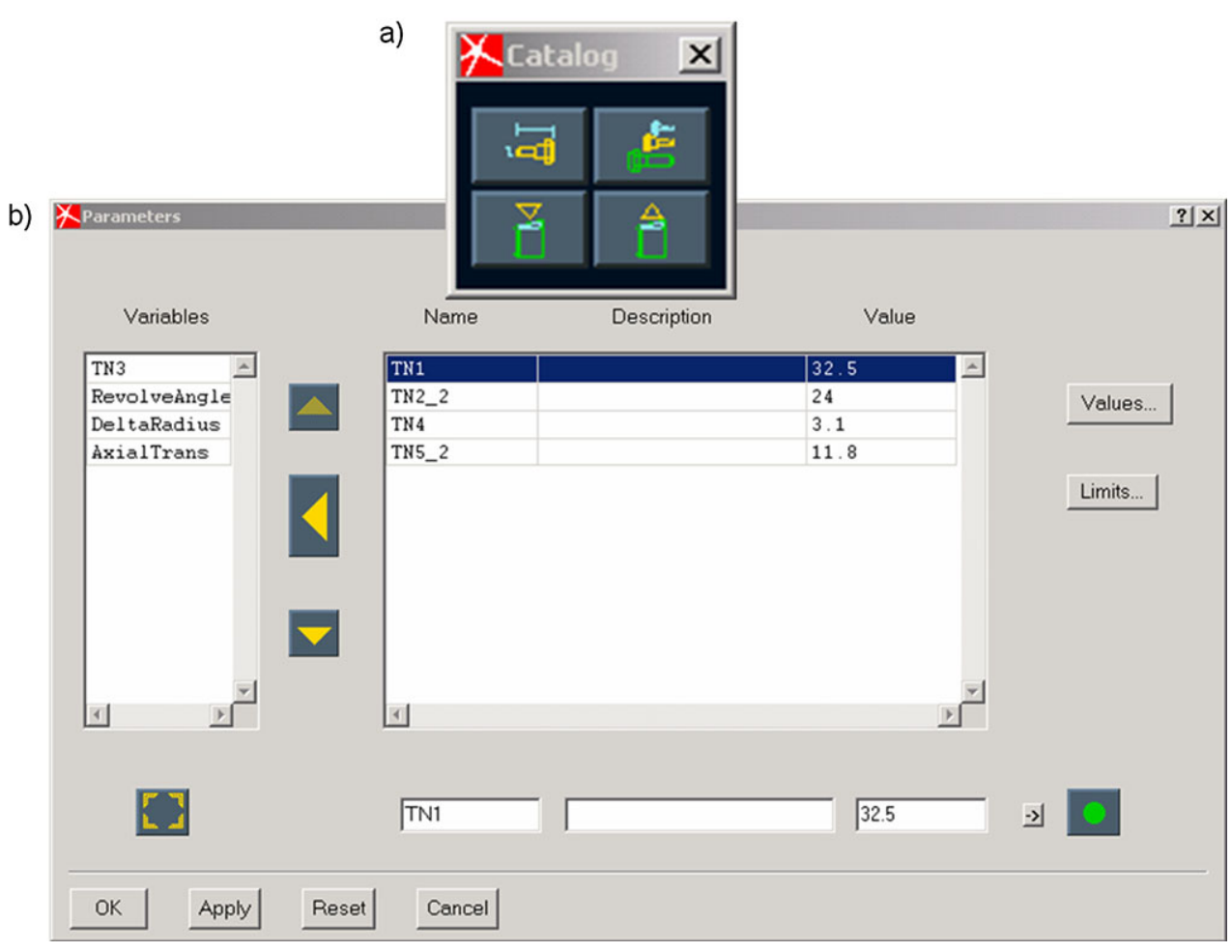

a) Abmessungswerteberechnung nach Ähnlichkeitsgesetze

b)

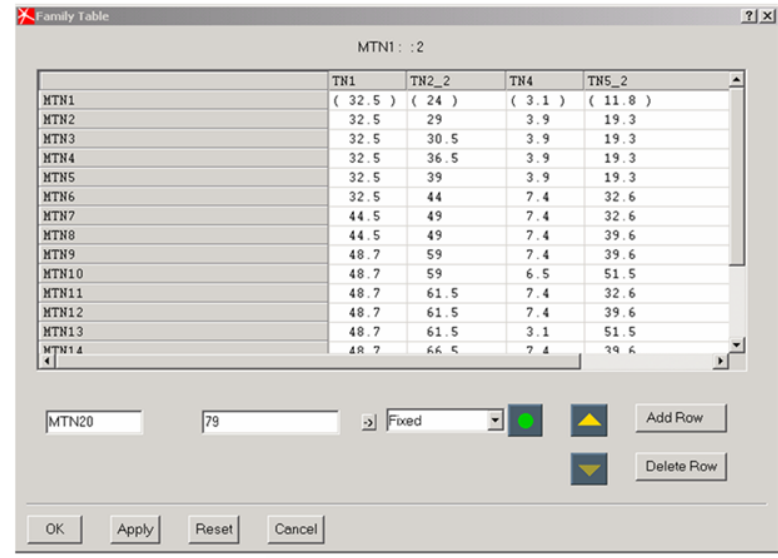
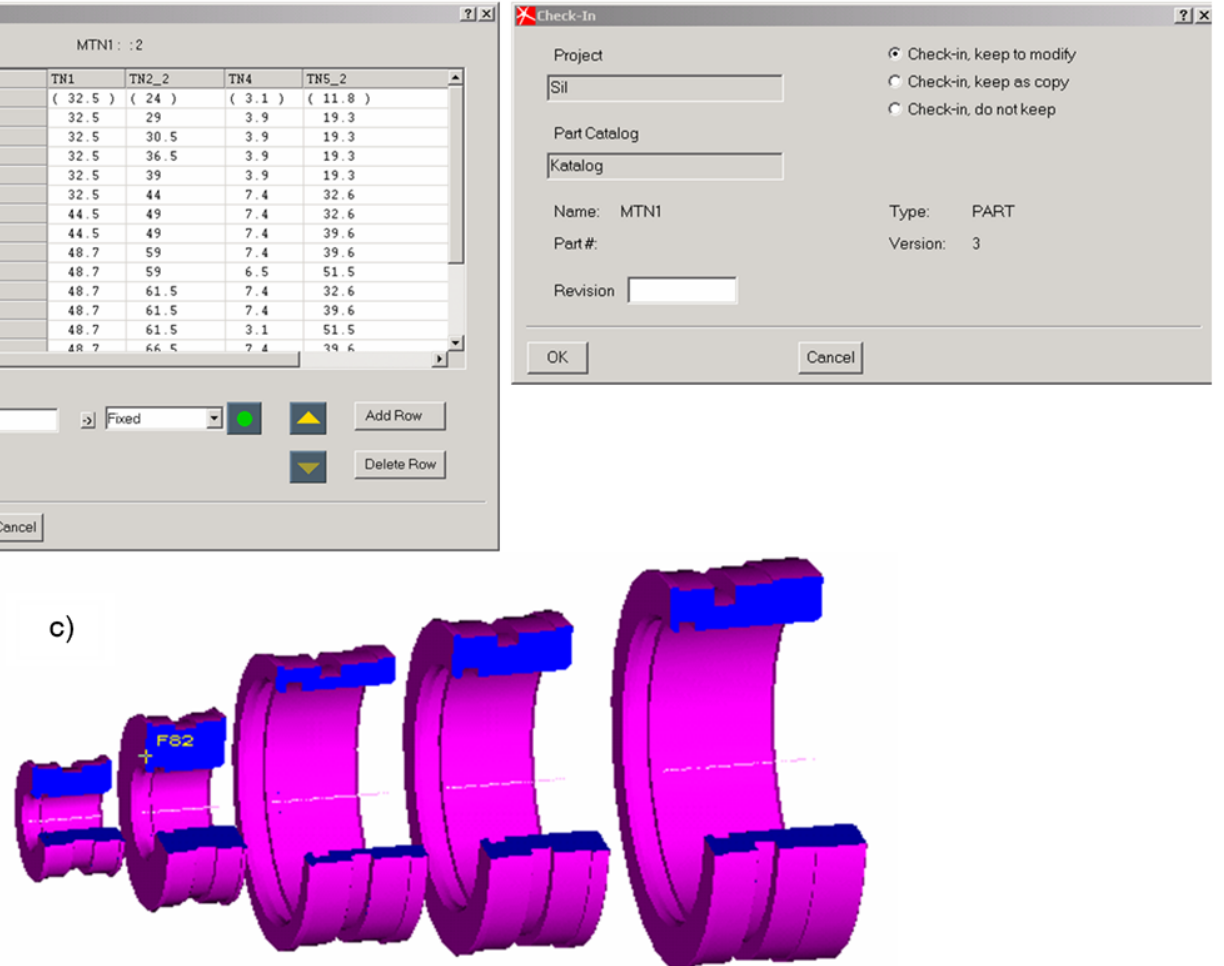

Abb. 11 Grafische Parametrisierung im fortgeschrittenem grafischen Progamm I-DEAS NX

gewählt werden (Abb. 10b). Variable Abmessungen sind Abmessungen deren Werte, zusammen mit einer Änderung der Abmessungsparameter von Konstruktionsfamilien, variieren.
Denn im Editor, unter den Bedingungen der Konstruktionsähnlichkeit, gewählten Abmessungen werden Abmessungswerte für jede Typengröße zugeordnet. Jede Typengröße erhält eine eigene Identifikationsnummer (Abb. 11a). 
Abb. 12 Aufzeichnung einer Typenreihe auf der Basis der grafischen Parametrisierung im NX Programm

Abb. 13 Aufzeichnung einer Typenreihe mittels relationaler Parametrisierung im I-DEAS NX

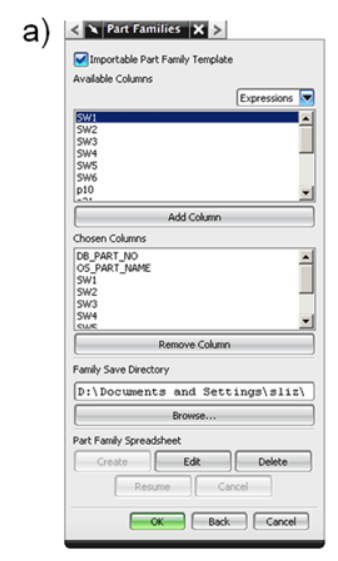

c)

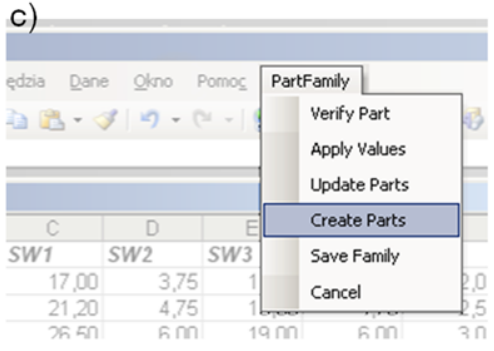

b) d)

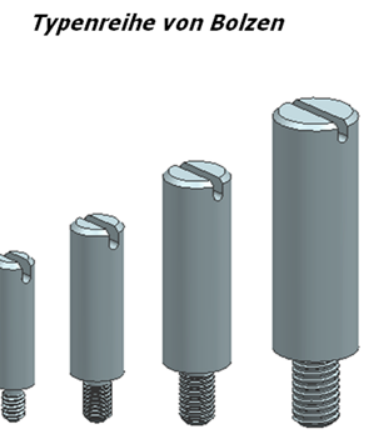

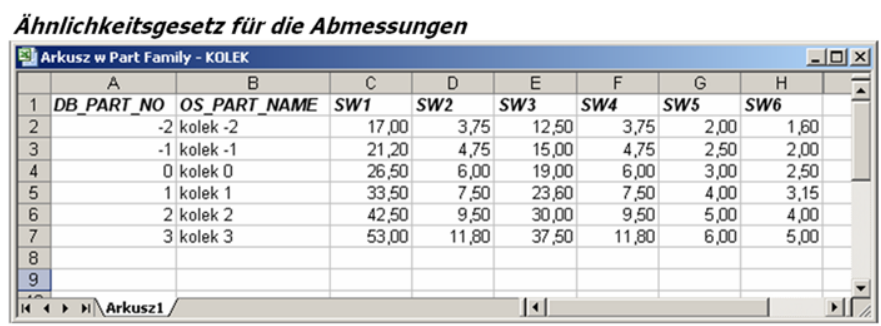
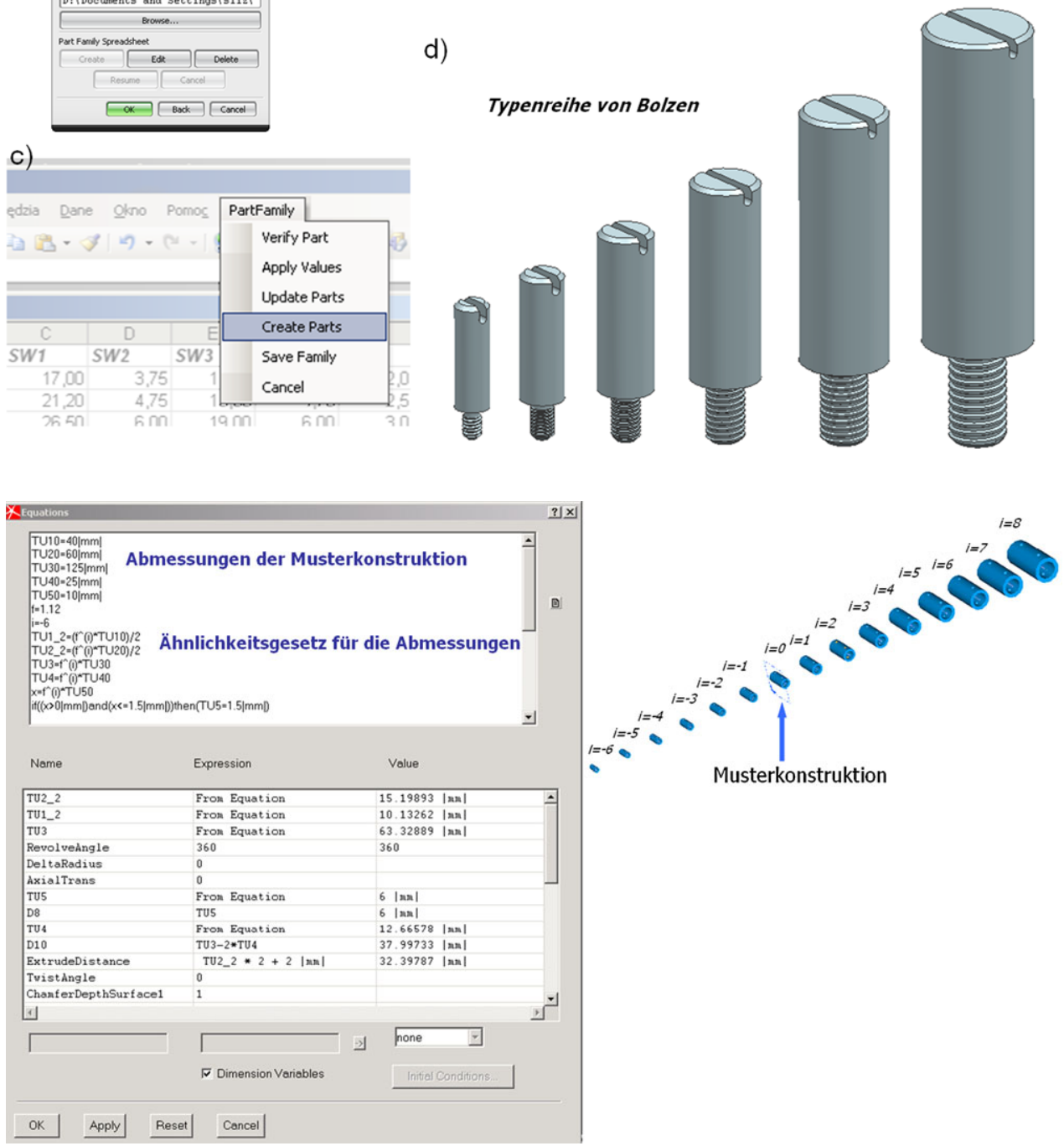

Ein auf diese Weise parametrisiertes 3D-Modell wird in das Verzeichnis der Typenreihe einer Konstruktionsfamilie übernommen (Abb. 11b).

Auf der Grundlage der grafischen Parametrisierung werden aus dem Verzeichnis einzelne Typengrößen von 3D-Elementen ausgewählt mit dem Ziel Baugruppen für künftige technische Mittel zu erstellen. Ein ähnlicher Parametrisierungsprozess wird im fortgeschrittenen grafischen Programm NX durchgeführt. Nach der Auswahl der variablen Abmessungen des Mustermodells im Programmmodul „Part Familes“ (Abb. 12a) wird, entsprechend der Be- dingungen der Konstruktionsähnlichkeit, die Auswahl zu den Abmessungswerten in einem Excel-Formular getroffen (Abb. 12b). Nach der Übernahme eines auf diese Weise parametrisierten Modells in das Verzeichnis (Abb. 12c), besteht die Möglichkeit der Verwendung dessen erzeugten Typengrößen.

Relationale Parametrisierung verwendet einen internen Formeleditor des grafischen Programms. Im Editor werden die variablen Abmessungswerte der Musterkonstruktion bestimmt und danach in Abhängigkeit von der Ähnlichkeitszahl für die Abmessungen $\varphi_{l}$, die Bedingungen der Kon- 
struktionsähnlichkeit aufgezeichnet (Abb. 13a). Um ein bestimmtes 3D-Modell einer Typengröße im Editor zu bekommen wird ein bestimmter Wert der Variablen $i$ zugeordnet (z. B.: $i=-6,8$ ). Wobei das Modell der Musterkonstruktion eine Bezeichnung $i=0$ besitzt. Beispielhafte 3DModelle einer Hülse, erzeugt auf der Basis der relationalen Parametrisierung, sind in der Abb. 13b dargestellt.

Die besten Parametrisierungseffekte werden im Hinblick auf die Möglichkeit der Integration der Auswahl von Konstruktionsmerkmalen mit der Verwendung der programmierbaren Parametrisierung erzielt. Auf dieser Grundlage werden in Rahmen von Projekten geordnete Konstruktionsfamilien von Nadelgreifern und pneumatischen Greifern gebildet.

\section{Schlussbemerkung}

Die durchgeführten Untersuchungen zeigen, dass durch Ähnlichkeitsgesetze und Musterkonstruktion eine optimale Typenreihe gebildet werden können. Weiterhin bilden die gleichen, wie in der Musterkonstruktion vorhanden, physikalischen, stereomechanischen und einfachen Zustände die Grundlage für Ähnlichkeitsgesetze von Konstruktionsfamilien. Die Aufzeichnung derartig gebildeter Typenreihen ist mit programmierbarer, graphischer und relationaler Parametrisierung möglich. Die Ähnlichkeitsgesetze werden bereits experimentell, an entwickelten Typenreihen, verifiziert. Deren Beschreibung folgt in der nächsten Publikation.
Danksagung Gebildet auf Grund des DAAD Stipendiums in TU Chemnitz (1.08.12-30.09.12) und im Rahmen des Forschungsprojektes N502 087638.

Open Access This article is distributed under the terms of the Creative Commons Attribution License which permits any use, distribution, and reproduction in any medium, provided the original author(s) and the source are credited.

\section{Literatur}

1. Gendarz P (1998) Berechnung von Abmessungen für die Entwicklung von Baureihen. Konstruktion 10.98:23-28

2. Gendarz P (2004) Methoden und Hauptzuordnungen im Prozess der Bildung von geordneten Konstruktionsfamilien. In: 15 Symposium "Design for X". The Design Society a worldwide community, Neukirchen, S 95-104. 2004

3. Gendarz P (2009) Hauptzuordnungen bei Modulbildungsprozessen von Konstruktionen. Forsch Ingenieurwesen Eng Res 73:245-255

4. Gendarz P (2010) Kopplungsrelationen im Prozess der Bildung geordneter Konstruktionsfamilien. Forsch Ingenieurwesen Eng Res 74:175-184

5. Gendarz P, Cielniak M (2012) Quantitative constructional attributes selection in construction series of types. J Achiev Mater Manuf Eng 49(1):62-70

6. Gerhard E (1984) Baureihenentwicklung. Kontakt \& Studium Band, Bd 105. Expert Verlag, Grafenaut

7. Pahl G, Beitz WJ, Feldhusen K, Grote H (2006) Pahl/Beitz Konstruktionslehre. Grundlagen. Springer, Berlin 